Research article

Open Access

\title{
Evaluation of unclassified variants in the breast cancer susceptibility genes BRCA1 and BRCA2 using five methods: results from a population-based study of young breast cancer patients
}

\author{
Eunjung Lee1, Roberta McKean-Cowdin¹, Huiyan Ma², Zhengjia Chen¹,3, David Van Den Berg1, \\ Brian E Henderson ${ }^{1}$, Leslie Bernstein ${ }^{4}$ and Giske Ursin ${ }^{1,5}$
}

\author{
1Department of Preventive Medicine, Keck School of Medicine, University of Southern California, 1441 Eastlake Avenue, USC/Norris Comprehensive \\ Cancer Center, Los Angeles, CA 90089-9175, USA \\ 2Institute for Health Promotion and Disease Prevention Research, Keck School of Medicine, University of Southern California, Alhambra, CA 91803, \\ USA \\ ${ }^{3}$ Children's Oncology Group, Arcadia, CA 91066, USA \\ ${ }^{4}$ City of Hope Comprehensive Cancer Center and Beckman Research Institute, Duarte, CA 91010, USA \\ ${ }^{5}$ Department of Nutrition, University of Oslo, Norway \\ Corresponding author: Giske Ursin, gursin@usc.edu
}

Received: 7 Nov 2007 Revisions requested: 8 Jan 2008 Revisions received: 20 Jan 2008 Accepted: 19 Feb 2008 Published: 19 Feb 2008

Breast Cancer Research 2008, 10:R19 (doi:10.1186/bcr1865)

This article is online at: http://breast-cancer-research.com/content/10/1/R19

(c) 2008 Lee et al.; licensee BioMed Central Ltd.

This is an open access article distributed under the terms of the Creative Commons Attribution License (http://creativecommons.org/licenses/by/2.0), which permits unrestricted use, distribution, and reproduction in any medium, provided the original work is properly cited.

\begin{abstract}
Introduction Efforts are ongoing to determine the significance of unclassified variants (UVs) in the breast cancer susceptibility genes $B R C A 1 / B R C A 2$, but no study has systematically assessed whether women carrying the suspected deleterious UVs have characteristics commonly seen among women carrying known deleterious or disease-causing mutations in $B R C A 1 / B R C A 2$.

Methods We sequenced $B R C A 1 / B R C A 2$ in 1,469 populationbased female breast cancer patients diagnosed between the ages of 20 and 49 years. We used existing literature to classify variants into known deleterious mutations, polymorphic variants, and UVs. The UVs were further classified as high risk or low risk based on five methods: allele frequency, Polyphen algorithm, sequence conservation, Grantham matrix scores, and a combination of the Grantham matrix score and sequence conservation. Furthermore, we examined whether patients who carry the variants classified as high risk using these methods have risk characteristics similar to patients with known deleterious $B R C A 1 / B R C A 2$ mutations (early age at diagnosis, family history of breast cancer or ovarian cancer, and negative estrogen receptor/progesterone receptor).
\end{abstract}

Results We identified 262 distinct $B R C A 1 / B R C A 2$ variants, including $147 \mathrm{UVs}$, in our study population. The $B R C A 1 \mathrm{UV}$ carriers, but not the BRCA2 UV carriers, who were classified as high risk using each classification method were more similar to the deleterious mutation carriers with respect to family history than those carriers classified as low risk. For example, the odds ratio of having a first-degree family history for the high-risk women classified using Polyphen was 3.39 (95\% confidence interval $=1.16$ to 9.94$)$ compared with normal/polymorphic BRCA1 carriers. The corresponding odds ratio of low-risk women was 1.53 (95\% confidence interval $=1.07$ to 2.18 ). The odds ratio for high-risk women defined by allele frequency was 2.00 (95\% confidence interval $=1.14$ to 3.51$)$, and that of lowrisk women was 1.30 (95\% confidence interval $=0.87$ to 1.93 ).

Conclusion The results suggest that the five classification methods yielded similar results. Polyphen was particularly better at isolating $B R C A 1 \mathrm{UV}$ carriers likely to have a family history of breast cancer or ovarian cancer, and may therefore help to classify BRCA1 UVs. Our study suggests that these methods may not be as successful in classifying BRCA2 UVs.

$\mathrm{BIC}=$ Breast Cancer Information Core; DDCV = definitely disease-causing variant; $\mathrm{ER}=$ estrogen receptor; GMS = Grantham matrix score; HFUV $=$ high-frequency unclassified variant; LFUV $=$ low-frequency unclassified variant; $P R=$ progesterone receptor; UV $=$ unclassified variant. 


\section{Introduction}

In the early 1990s the breast cancer susceptibility genes $B R C A 1$ and $B R C A 2$ were identified through linkage analyses [1-4]. BRCA1, located on chromosome 17q12-q21, consists of 24 exons encoding a protein of 1,863 amino acids and is involved in DNA repair [5,6], in transcription [7,8], and in the cell cycle checkpoint in DNA damage response [9-11]. $B R C A 2$, located on chromosome 13q12-q13, consists of 27 exons encoding a protein of 3,418 amino acids and is also involved in DNA repair [12-15], but its role in transcription and the cell cycle checkpoint is less clear [16].

Since the discovery of the BRCA1 and BRCA2 genes, a total of 1,643 and 1,856 distinct variants have been reported in the Breast Cancer Information Core (BIC) Database for BRCA1 and BRCA2 as of April 2007 [17]. Among these variants, frameshift mutations, nonsense mutations, splice variants and a few well-documented missense mutations are considered deleterious [18], while synonymous variants have been considered benign or polymorphic. A large number of missense or intronic variants of $B R C A 1$ or $B R C A 2$ remain of unknown significance. The proportion of breast cancer patients who carry these unclassified variants (UVs) is about 9\% [19]. Given that only $2 \%$ to $3 \%$ of breast cancer patients have deleterious mutations in BRCA1 or BRCA2 [20], understanding the clinical significance of this relatively large number of UVs is of great importance.

Functional studies can provide direct insight into whether the UV has biological consequences, but few of these studies have been performed $[21,22]$. Other approaches have been applied to classify the significance of UVs, including comparisons of allele frequencies [18], algorithms such as Polyphen (see Materials and methods) [23], examination of sequence conservation across species [24-26], and characterization of the physicochemical nature of the amino acid substitutions (Grantham matrix scores) $[26,27]$. A combination approach of the sequence conservation and Grantham matrix score methods was applied to classify a large number of UVs [26]. No systematic evaluation, however, has been conducted to determine whether patients who carry the variants classified as high risk using these methods have similar characteristics as patients with known deleterious BRCA1/BRCA2 mutations, which would suggest that these high-risk UVs are deleterious.

Breast cancer patients with a known deleterious mutation in $B R C A 1 / B R C A 2$ are more likely to have a family history of breast cancer or ovarian cancer [28] and an earlier age of diagnosis than noncarrier patients $[18,29]$. In addition, $B R C A 1$ deleterious mutation carriers are more likely to have estrogen receptor (ER)-negative and progesterone receptor (PR)-negative tumors than women without such mutations [29]. In the current analyses, we classified BRCA1/BRCA2 UVs using the four methods listed above and a combination of the Grantham matrix scores and sequence conservation. We then evaluated the validity and usefulness of each method by comparing the risk categories of UV carriers with respect to these three well-defined characteristics of BRCA1/BRCA2 deleterious mutation carriers.

\section{Materials and methods Subjects}

The data collection methods for this study have been described previously [30]. In brief, female patients diagnosed with histologically confirmed first primary invasive breast cancer were identified through the Los Angeles County Cancer Surveillance Program, a population-based Surveillance, Epidemiology and End Results registry supported by the State of California and the National Cancer Institute. Eligible cases were US born and English speaking, white (including Hispanic) or African-American, aged 20 to 49 years at diagnosis, and Los Angeles County residents at diagnosis. A total of 2,882 eligible cases were identified (2,534 whites and 348 African-Americans) between February 1998 and May 2003. Recruitment of African-Americans began after the initiation of the study with eligible African-American cases diagnosed from January 2000 to May 2003.

Among the 2,882 potentially eligible cases, 1,794 (62\%) were interviewed (1,585 white, 209 African-American). Reasons for nonparticipation were patient refusal $(n=428)$, no longer a resident of Los Angeles County $(n=37)$, not located $(n=88)$, death $(n=38)$, serious illness or disability $(n=18)$, physician refusal $(n=50)$, or inability to schedule the interview within 18 months of diagnosis $(n=429)$. The study was approved by the Institutional Review Board of the University of Southern California. All participants provided written informed consent.

\section{Data and blood specimen collection}

An inperson interview was completed using a modified version of the structured questionnaire used in the Women's Contraceptive and Reproductive Experiences Study [31]. The questionnaire included detailed information on demographic characteristics, family history of breast cancer or ovarian cancer, ethnic origin, and environmental factors such as oral contraceptive use, reproductive history, alcohol use, smoking history, and radiation exposure. We obtained information up to the date of breast cancer diagnosis. Blood specimens were collected from 1,519 participants (85\%) and were transported to the Norris Cancer Center Genetics Core Laboratory in Styrofoam containers on frozen ice packs. For the first 50 samples the buffy coat was immediately extracted and stored, and for the remaining samples we stored whole blood.

\section{Sequencing of BRCA1 and BRCA2 genes}

All $B R C A 1$ and $B R C A 2$ exons (except BRCA1 exons 1 and 4 and $B R C A 2$ exon 1) as well as all exon-intron boundaries were sequenced. Exon 1 was not sequenced for either gene because it is located upstream of the translation start site in 
both genes. BRCA1 exon 4 was not sequenced because it is not found in the normal BRCA1 mRNA transcript.

DNA extraction, amplification and sequencing were carried out in the USC Genomics Core Laboratory using a protocol similar to that previously described [32]. The detailed procedures are described in the supplemental methods (see Additional File 1). We sequenced BRCA1/BRCA2 genes for 1,469 out of 1,519 blood specimens. We were unable to sequence the remaining 50 specimens due to insufficient DNA.

Thirty-three randomly selected, blinded samples were resequenced for quality control purposes. The discordance rate was $0.19 \%$ : 16 discordant sequencing results out of the total 8,646 variant sites sequenced (262 variant sites for each of the 33 samples). In addition, 166 subjects who had noninformative sequencing results on one or more variant sites were resequenced or genotyped using the TaqMan assay (for BRCA2 I2490T, N372H, and N991D) as previously described [33].

\section{Epidemiologic and histologic variables}

Age at diagnosis was categorized as $<35$ years, 35 to 39 years, 40 to 44 years, and 45 to 49 years. We classified women based on their family history of breast cancer or ovarian cancer as follows: one or more breast cancer or ovarian cancer patients among their first-degree relatives (mother and full sisters); no first-degree family history of breast cancer or ovarian cancer but one or more breast cancer or ovarian cancer patients among their second-degree relatives (mother's or father's full sisters, and grandmothers); no first-degree or second-degree relatives diagnosed with breast cancer or ovarian cancer; and an unknown first-degree family history. We considered unknown second-degree family history as no family history.

The ER and PR status of the breast cancer was obtained by abstracting pathology reports collected by the Los Angeles County Cancer Surveillance Program. Among the 1,469 subjects, ER/PR information was available for 1,216 patients (83\%). For the ER/PR analyses, we excluded 63 patients who had borderline ER/PR status and 101 patients whose ER/PR status was $+/-$ or $-/+$, leaving 1,052 patients with a $+/+$ or $-/-$ receptor status.

\section{Classification of BRCA1/BRCA2 mutation status}

We classified each identified $B R C A 1 / B R C A 2$ variant according to its predicted functional and biological significance as follows: definitely disease-causing variants (DDCVs), including frameshift mutations, nonsense mutations, splice variants that were previously reported to affect splicing or were located at the exon/intron boundary, and missense variants that were previously shown to be deleterious; UVs, including inframe deletion/insertions, intronic variants that might affect splicing by creating a splice donor/acceptor site, variants next to the exon/intron boundary, and most missense variants; and benign polymorphic variants, including synonymous variants, intronic variants that are unlikely to affect splicing, and a few missense mutations that were reported to be benign. (See Additional File 2 for a list of all variants identified in this study, with their classification and the reasons and references for such classification.)

\section{Further classification of BRCA1 and BRCA2 unclassified variants}

We further classified $B R C A 1 / B R C A 2$ UVs using the following methods.

\section{Classification based on allele frequency}

We divided the UVs into high-frequency unclassified variants (HFUVs) and low-frequency unclassified variants (LFUVs) depending on the minor allele frequency $(\geq 1 \%$ versus $<1 \%$ ) in each ethnic group (142 African-Americans, 222 Hispanic whites, 1,105 non-Hispanic whites). If the minor allele frequency is $\geq 1 \%$ in one or more ethnic groups, the UV was categorized as a HFUV. This categorization was based on the assumption that variants with high frequency would be less likely to be disease causing compared with variants of very low frequency.

\section{Polyphen-based classification}

Polyphen is an algorithm that classifies the functional effect of each missense variant into three categories (probably damaging, possibly damaging, and benign) [34]. This classification is based on the chemical characteristics of the substitution site (for example, disulfide bond, transmembrane region), the alignment of homologous sequences, and protein three-dimensional structures [23]. UVs other than missense variants are not classified by Polyphen. The Polyphen classification in this report is based on access to the algorithm in March 2007.

\section{Classification based on sequence conservation across mammalian species}

A variant that occurs at a site with high-degree conservation is considered more likely to be deleterious than a variant occurring at a site with low-degree conservation [35]. We selected only mammals for cross-species comparisons of the BRCA1/ $B R C A 2$ sequences, since the function of these two proteins in mammals could be different from that in other animals. We selected all mammalian species whose BRCA1/BRCA2 sequences were reported in the National Center for Biotechnology Information gene database or whose complete coding sequences were reported in the National Center for Biotechnology Information nucleotide sequence database. Ten species for $B R C A 1$ and five species for $B R C A 2$ met these criteria (see Additional File 2). Sequence alignment was performed using the Clustal W method [36] and the MegAlign software (DNASTAR, Inc., Madison, WI, USA). 
We classified BRCA1/BRCA2 missense variants into three categories (high conservation, moderate conservation, and low conservation) depending on the number of the species that had a different amino acid from that of the human at the site of variation. For each UV in BRCA1 we considered differences in zero or one species out of the 10 examined to represent high conservation, differences in two or three species to represent moderate conservation, and differences in four or more species to represent low conservation. For BRCA2 we compared sequences of five species: no difference in all five species was considered high conservation, one or two differences were considered moderate conservation, and three or more differences were considered low conservation.

\section{Classification based on the Grantham matrix score}

The Grantham matrix score (GMS) is a composite measure of the degree of amino acid substitution, taking into account the side-chain composition, polarity, and molecular volume of the two amino acids [27]. We dichotomized the GMS at 60, a criterion previously used to define neutral missense variants [26].

\section{Integration of sequence conservation and the Grantham} matrix score

We adopted a previously reported classification scheme integrating the sequence conservation and the GMS [26]. Briefly, if the variant was located at a fully conserved site or led to a nonconservative substitution at a conserved site, it was considered deleterious. If the variant amino acid is observed in other species or led to conservative substitution, it was considered neutral. See Additional File 1 for further details.

\section{Classification of women who carry unclassified variants in BRCA1/BRCA2}

Each subject was categorized hierarchically based on their $B R C A 1$ and BRCA2 mutation status (Figure 1). This means that anyone successfully classified by the first criterion would not be further classified by the criteria that followed. This hierarchical classification leads to mutually exclusive categories (DDCV carriers, UV carriers, normal/polymorphic carriers, and patients with unknown mutation status) as follows. First, a patient was classified as a DDCV carrier if she had one or more of the DDCV(s). Second, if the patient did not belong to the DDCV group and had a noninformative result at any of the identified DDCV sites, she was classified as unknown. Third, if the patient did not belong to these first two categories and carried one or more of the UVs, she was classified as a UV carrier. Fourth, if the patient did not belong to the first three categories and any of the sequencing results at the identified UV sites was noninformative for the subject, she was classified as unknown. Finally, if the patient did not belong to any of the preceding categories, she was classified as a polymorphic or normal genotype carrier.

UV carriers were further classified hierarchically into mutually exclusive categories of high risk, moderate risk, low risk, and unknown risk according to the various UV classifications. For example, when applying the allele frequency method, a UV carrier was classified as high risk if the subject carried one or more of the LFUVs, as unknown risk if any of the sequencing results at the LFUV site was noninformative for the subject, as low risk if the subject carried one or more of the HFUVs, and as unknown risk if any of the sequencing results at the HFUV site was noninformative for the subject. Classification using

Figure 1

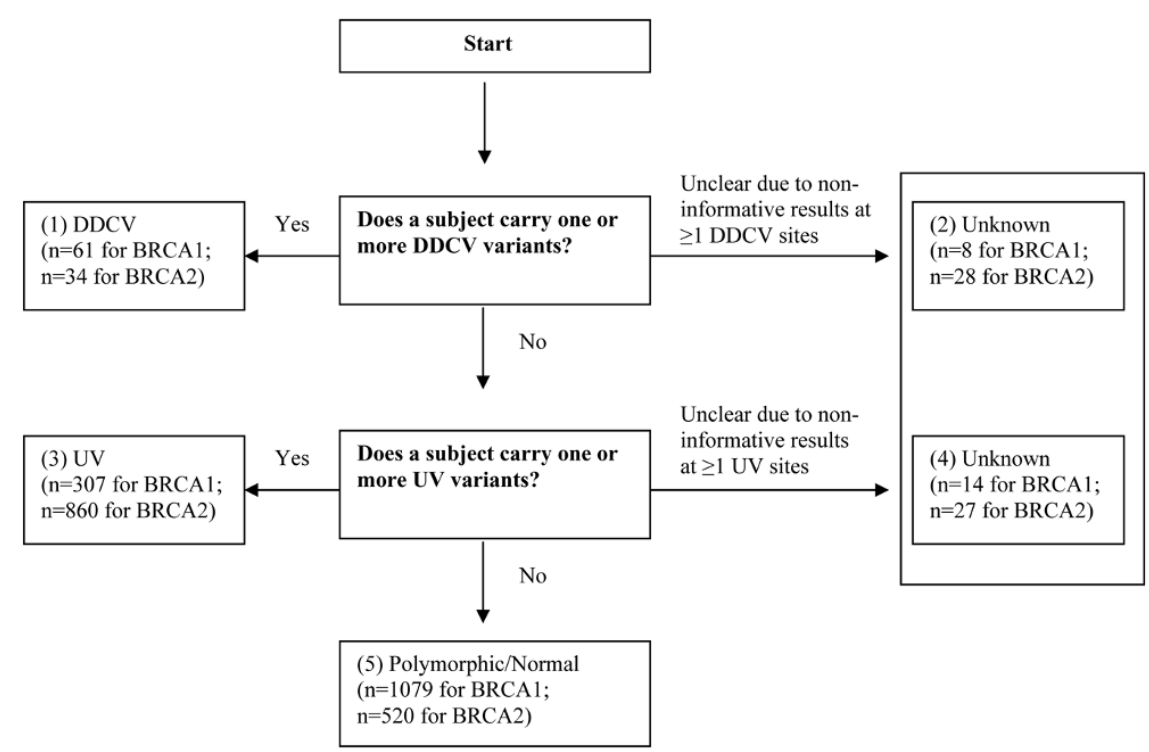

Illustration of the classification scheme of $B R C A 1 / B R C A 2$ variants. DDCV, definitely disease-causing variant; UV, unclassified variant. 
other methods such as Polyphen, the GMS, or sequence conservation followed the same hierarchical logic.

Six BRCA1 UV carriers and six BRCA2 UV carriers with a possible splice variant or in-frame deletion were categorized only by allele frequency since Polyphen, the GMS, and the integrated GMS/sequence conservation methods are not applicable to these splice variants and in-frame deletions. These women were therefore excluded from the analyses using Polyphen, the GMS, sequence conservation, and the integrated GMS/sequence conservation methods.

\section{Statistical analyses}

We compared the UV classification methods of allele frequency, Polyphen, sequence conservation, and the GMS by examining the pairwise joint distribution of BRCA1/BRCA2 UVs as classified using each method. Tests for a linear trend in the GMS across the three UV categories classified using Polyphen and the sequence conservation method were conducted in linear regression models. The mean GMS across two UV categories using allele frequency was compared by $t$ test. We assessed whether UV classifications using allele frequency, Polyphen and the sequence conservation methods are correlated using an exact Mantel-Haenszel chi-square test.

We performed case-case analyses to examine the association between BRCA1 or BRCA2 carrier status categorized using each method (exposure variable) and outcome variables (clinical and disease characteristics). Case-case analyses were conducted using polychotomous logistic regression when the outcome variable was family history of breast cancer or ovarian cancer. The association with the ER/PR status was analyzed using logistic regression. We used linear regression where the outcome variable was age at diagnosis of breast cancer. When examining $B R C A 1$, results were adjusted for the BRCA2 mutation status (DDCV, non-DDCV, unknown), and vice versa.

All reported $P$ values are two-sided. The SAS 9.1 package was used for all analyses (SAS Institute, Cary, NC, USA).

\section{Results}

A total of 105 distinct $B R C A 1$ variants (including $32 \mathrm{DDCVs}$ ) and 157 distinct $B R C A 2$ variants (including $27 \mathrm{DDCVs}$ ) were identified in the 1,469 breast cancer patients (see Additional File 3). Among these distinct variants, 22 BRCA 1 variants and $30 B R C A 2$ variants had not been reported in the $\mathrm{BIC}$ as of April 2007.

\section{Correlated classifications using various approaches}

Classification using the Polyphen algorithm appeared to be correlated both with the GMS and the conservation method: $B R C A 1 / B R C A 2$ missense variants classified as high risk (probably damaging) using Polyphen had a higher mean GMS than those classified as low risk (benign missense variants) (Table 1). BRCA1/BRCA2 missense variants classified as benign missense variants using Polyphen were generally located at sites with low degree of sequence conservation, while probably damaging missense variants tended to be located in highly conserved regions (Table 2). The GMS, however, was not strongly correlated with level of conservation across species (Table 1). Given the small number of HFUVs of $B R C A 1 / B R C A 2$, the classification using the allele frequency method seemed to be associated with the classifications using other methods, although not all of these analyses achieved statistical significance.

\section{Classification of case patients with regard to $B R C A 1$ or BRCA2 status}

Among the 1,469 case patients in this study, 61 women carried a BRCA1 DDCV and 34 women carried a BRCA2 DDCV. Among the remaining women, 307 women and 860 women were UV carriers in BRCA1 and in BRCA2, respectively.

\section{Classification of BRCA1/BRCA2 status in relation to epidemiologic and histologic outcome variables}

Family history of breast cancer or ovarian cancer

The BRCA1 DDCV carriers were substantially more likely to have a first-degree family history of breast cancer or ovarian cancer than the normal/polymorphic BRCA1 carriers (odds ratio $=11.3$; Table 3 ) after adjusting for the $B R C A 2$ mutation status. The UV carriers were also significantly, although to a smaller extent, more likely to have a first-degree family history than normal/polymorphic BRCA1 carriers (odds ratio $=1.54$ ). The high-risk UV carriers were, in general, significantly more likely to have a first-degree family history of breast cancer or ovarian cancer than normal/polymorphic women, whereas the low-risk UV carriers were not. For example, the high-risk UV carriers identified using the allele frequency (LFUV) or Polyphen (probably damaging) methods were more likely to have a first-degree family history (odds ratio $=2.00$ and 3.39, respectively) than normal/polymorphic $B R C A 1$ carriers.

A similar trend was observed using the sequence conservation or the GMS method, although differences between the categories of UV carriers were smaller. The integrated method of the GMS/sequence conservation classified only nine subjects as high risk, and their odds ratio was not different from that of the women who remained unclassified.

The BRCA2 DDCV carriers were also at a higher risk of having a first-degree family history of breast cancer or ovarian cancer compared with the normal/polymorphic BRCA2 carriers (odds ratio $=3.69$ ) after adjusting for $B R C A 1$ mutation status. The association was weaker than that of $B R C A 1$ DDCV carriers. Regardless of the classification method, the high-risk UV carriers were not statistically significantly different from the normal/polymorphic BRCA2 carriers with regard to family history (Table 3). 
Table 1

Mean Grantham matrix score of BRCA1/BRCA2 variants (unclassified variants) according to classification using allele frequency, Polyphen, and sequence conservation

\begin{tabular}{|c|c|c|c|c|c|c|c|}
\hline \multirow[t]{2}{*}{ Gene } & \multirow[t]{2}{*}{ Classification method } & \multirow[t]{2}{*}{ Level } & \multirow[t]{2}{*}{ Number of variants } & \multicolumn{4}{|c|}{ Grantham matrix score } \\
\hline & & & & Mean & Standard deviation & Minimum & Maximum \\
\hline \multirow[t]{11}{*}{$B R C A 1$} & Allele frequency & Low risk (HFUV) & 5 & 38.2 & 20.7 & 10 & 56 \\
\hline & & High risk (LFUV) & 39 & 69.7 & 45.0 & 10 & 194 \\
\hline & & $P$ value ( $t$ test) & & 0.13 & & & \\
\hline & Polyphen & Benign missense & 28 & 55.0 & 42.3 & 10 & 180 \\
\hline & & Possible damaging & 9 & 65.3 & 29.4 & 10 & 101 \\
\hline & & Probably damaging & 7 & 111.9 & 40.5 & 71 & 194 \\
\hline & & $P$ for trend ${ }^{a}$ & & 0.002 & & & \\
\hline & Sequence conservation & Low & 14 & 53.6 & 44.7 & 10 & 180 \\
\hline & & Moderate & 14 & 70.9 & 43.5 & 10 & 154 \\
\hline & & High & 16 & 73.0 & 44.2 & 10 & 194 \\
\hline & & $P$ for trenda & & 0.24 & & & \\
\hline \multirow[t]{11}{*}{$B R C A 2$} & Allele frequency & Low risk (HFUV) & 18 & 67.7 & 39.7 & 5 & 149 \\
\hline & & High risk (LFUV) & 77 & 87.0 & 52.9 & 10 & 205 \\
\hline & & $P$ value $(t$ test $)$ & & 0.15 & & & \\
\hline & Polyphen & Benign missense & 44 & 58.1 & 41.3 & 5 & 194 \\
\hline & & Possible-damaging & 25 & 91.4 & 41.7 & 21 & 180 \\
\hline & & Probably-damaging & 26 & 118.3 & 52.3 & 27 & 205 \\
\hline & & $P$ for trend ${ }^{a}$ & & $<0.001$ & & & \\
\hline & Sequence conservation & Low & 33 & 71.5 & 49.4 & 10 & 194 \\
\hline & & Moderate & 29 & 92.1 & 55.6 & 5 & 205 \\
\hline & & High & 33 & 87.5 & 47.8 & 21 & 194 \\
\hline & & $P$ for trenda & & 0.20 & & & \\
\hline
\end{tabular}

LFUV, low-frequency unclassified variant; HFUV, high-frequency unclassified variant. aBased on the $F$ test in a linear regression model.

Age at diagnosis and estrogen receptor/progesterone receptor status

As expected, compared with the carriers of normal/polymorphic BRCA1, the BRCA1 DDCV carriers had a much earlier age at diagnosis (by 4.1 years; $P<0.001$ ) and more ER/PRnegative tumors (odds ratio $=7.24,95 \%$ confidence interval $=3.56$ to 14.7). Case patients with high-risk UVs, however, did not have such characteristics regardless of the method of UV classification. The BRCA2 DDCV or UV status was not associated with early age at diagnosis or with ER/PR negativity (data not shown).

\section{Comparisons of the classifications using the methods in this study and the Breast Cancer Information Core}

The recent update of the BIC includes the assessment of the clinical importance of each variant. This assessment is based on several criteria, including epidemiological, segregation, and co-occurrence data. Among the UVs in this study, one BRCA1
UV (IVS5-11T > G) was classified as clinically important whereas three BRCA1 UVs and 19 BRCA2 UVs were classified as clinically nonimportant. IVS5-11T $>$ G was classified as a high-risk UV using allele frequency (LFUV). Since this variant is not a missense variant, other methods were not applicable. Table 4 shows how each UV that was considered nonimportant in the BIC was classified by the five UV classification methods. The allele frequency and the GMS method classified a large number of variants as high risk that were considered nonimportant by the BIC, particularly for BRCA2. In contrast, Polyphen and the conservation methods classified few such variants as high risk.

\section{Discussion}

In the present study of young breast cancer patients, we identified numerous variants in BRCA1/BRCA2 by direct sequencing, including $22 B R C A 1$ and $30 B R C A 2$ new variants that have not been reported in the BIC as of April 2007. 
Table 2

Joint distribution of BRCA1/BRCA2 variants (unclassified variants) according to classification using allele frequency, Polyphen, and sequence conservation

\begin{tabular}{|c|c|c|c|c|c|c|c|}
\hline \multirow[t]{2}{*}{ Gene } & \multirow[t]{2}{*}{ Classification method } & \multirow[t]{2}{*}{ Level } & \multicolumn{3}{|c|}{ Conservation } & \multicolumn{2}{|c|}{ Frequency } \\
\hline & & & Low & Moderate & High & HFUV & LFUV \\
\hline \multirow[t]{7}{*}{$B R C A 1$} & Frequency & Low risk (HFUV) & 3 & 1 & 1 & & \\
\hline & & High risk (LFUV) & 11 & 13 & 15 & & \\
\hline & & $P$ value $^{\mathrm{a}}$ & & & 0.26 & & \\
\hline & Polyphen & Benign missense & 12 & 10 & 6 & 4 & 24 \\
\hline & & Possible damaging & 2 & 3 & 4 & 1 & 8 \\
\hline & & Probably damaging & 0 & 1 & 6 & 0 & 7 \\
\hline & & $P$ value $^{\mathrm{a}}$ & & & 0.002 & & 0.39 \\
\hline \multirow[t]{7}{*}{$B R C A 2$} & Frequency & Low risk (HFUV) & 10 & 6 & 2 & & \\
\hline & & High risk (LFUV) & 23 & 23 & 31 & & \\
\hline & & $P$ value $^{\mathrm{a}}$ & & & 0.018 & & \\
\hline & Polyphen & Benign missense & 26 & 11 & 7 & 11 & 33 \\
\hline & & Possible damaging & 5 & 10 & 10 & 5 & 20 \\
\hline & & Probably damaging & 2 & 8 & 16 & 2 & 24 \\
\hline & & $P$ value ${ }^{a}$ & & & $<0.001$ & & 0.089 \\
\hline
\end{tabular}

LFUV, low-frequency unclassified variant; HFUV, high-frequency unclassified variant. aBased on the exact Mantel-Haenszel chi-square test.

We applied various methods to classify 44 BRCA1 UVs and 95 BRCA2 UVs. To our knowledge, our study is the first to attempt to classify a large number of BRCA1/BRCA2 UVs identified in population-based breast cancer patients and to correlate these variants with outcome variables.

We found that classifications of BRCA1/BRCA2 UVs using the various classification methods in general agree with each other (Table 1 and Table 2). In particular, Polyphen seemed to be correlated with the GMS and with sequence conservation, which is expected given the composite nature of this algorithm. This intercorrelation supports the reliability of the classification methods.

In general, the BRCA1 UV carriers classified as high risk were at increased risk of having a family history of breast cancer or ovarian cancer. Family history has been considered a powerful tool in classifying UVs [37], and having a first-degree relative with breast cancer increases the breast cancer risk about twofold [38]. The odds ratio for the high-risk UV group was highest when using Polyphen, suggesting that the algorithm is better for the purpose of describing high-risk variants when using family history as a measure of true risk. We cannot exclude, however, the possibility that more stringent cutoff points to define the high-risk group using other methods (that is, high-degree conservation defined as no cross-species variation; or high GMS defined as >100) might increase the odds ratio estimates of the high-risk group. In this study, we did not have sufficient numbers of UV carriers to investigate this possibility.

Considering that the high-risk BRCA1 UV carriers classified using all of the classification methods were at a higher risk of having a family cancer history (either statistically significantly or nonsignificantly), we expected to observe similar trends using age of diagnosis or the ER/PR status as the outcome variables. This observation, however, did not occur. The narrow age range of our study subjects, all of whom were under age 50 at diagnosis, could have limited the study power. For analyses of the ER/PR status, our exclusion of about $30 \%$ of women because of missing, borderline, or mixed (-/+ or $+/-)$ ER/PR status may have limited the statistical power. Alternatively, it is possible that only truncating mutations (resulting in a complete loss of $B R C A 1$ functions), but not missense variants (retaining part of its ability; for example, the ability to interact with certain proteins), of BRCA1 lead to the high density of ER/PR-negative tumors.

For BRCA2, it is unclear why none of the classification methods identified high-risk UV carriers when family history was used as the measure of true risk. One explanation could be the fact that BRCA2 DDCV carriers themselves did not have such a high odds ratio as seen for BRCA1 DDCV carriers. The $B R C A 2 \mathrm{DDCV}$ carrier status was also not associated with age at diagnosis in this study, again possibly because all of our subjects were younger than 50 years and the age at diagnosis 
Breast Cancer Research Vol 10 No 1 Lee et al.

Table 3

Association between family history of breast cancer or ovarian cancer and BRCA1 or BRCA2 status of the breast cancer patients

\begin{tabular}{|c|c|c|c|c|c|c|c|}
\hline \multirow[t]{2}{*}{ Mutation/unclassified variant status } & \multirow{2}{*}{$\frac{\text { None }}{n^{\mathrm{a}}}$} & \multicolumn{3}{|c|}{ First degree } & \multicolumn{3}{|c|}{ Second degree } \\
\hline & & $n^{\mathrm{a}}$ & OR $(95 \% \mathrm{Cl})^{\mathrm{b}}$ & $P$ value ${ }^{c}$ & $n^{\mathrm{a}}$ & OR $(95 \% \mathrm{Cl})^{\mathrm{b}}$ & $P$ value $^{c}$ \\
\hline \multicolumn{8}{|l|}{$B R C A 1^{\mathrm{d}}$} \\
\hline Normal/polymorphism (reference) & 600 & 166 & 1 & & 282 & 1 & \\
\hline Definitely disease-causing variant & 13 & 35 & 11.3 (5.73 to 22.5$)$ & $<0.001$ & 11 & 1.89 (0.83 to 4.31$)$ & 0.13 \\
\hline Unclassified variant & 156 & 67 & 1.54 (1.10 to 2.15$)$ & 0.012 & 75 & $1.02(0.74$ to 1.39$)$ & 0.90 \\
\hline \multicolumn{8}{|l|}{ Unclassified variant classification using } \\
\hline \multicolumn{8}{|l|}{ Allele frequency } \\
\hline High risk (LFUV) & 39 & 21 & $2.00(1.14$ to 3.51$)$ & 0.016 & 22 & $1.20(0.70$ to 2.06$)$ & 0.52 \\
\hline Low risk (HFUV) & 115 & 42 & $1.30(0.87$ to 1.93$)$ & 0.20 & 52 & $0.96(0.67$ to 1.37$)$ & 0.82 \\
\hline \multicolumn{8}{|l|}{ Polyphene } \\
\hline Probably damaging missense & 7 & 7 & $3.39(1.16$ to 9.94$)$ & 0.026 & 6 & $1.80(0.60$ to 5.40$)$ & 0.30 \\
\hline Possibly damaging missense & 9 & 1 & 0.44 (0.06 to 3.54$)$ & 0.44 & 5 & $1.19(0.40$ to 3.60$)$ & 0.75 \\
\hline Benign missense & 133 & 57 & 1.53 (1.07 to 2.18$)$ & 0.021 & 61 & 0.97 (0.70 to 1.36$)$ & 0.87 \\
\hline \multicolumn{8}{|l|}{ Sequence conservatione } \\
\hline High or moderate ${ }^{f}$ & 50 & 23 & 1.68 (0.99 to 2.85$)$ & 0.053 & 32 & 1.35 (0.85 to 2.16$)$ & 0.20 \\
\hline High & 6 & 3 & $1.88(0.46$ to 7.63$)$ & 0.38 & 8 & 2.85 (0.98 to 8.30$)$ & 0.055 \\
\hline Moderate & 44 & 20 & 1.66 (0.95 to 2.91$)$ & 0.076 & 23 & $1.10(0.65$ to 1.86$)$ & 0.72 \\
\hline Low & 99 & 40 & 1.43 (0.95 to 2.16$)$ & 0.085 & 41 & $0.88(0.60$ to 1.31$)$ & 0.53 \\
\hline \multicolumn{8}{|l|}{ Grantham matrix score } \\
\hline High $(>60)$ & 15 & 10 & 2.38 (1.04 to 5.45$)$ & 0.039 & 10 & 1.42 (0.63 to 3.20$)$ & 0.40 \\
\hline Low $(\leq 60)$ & 134 & 55 & 1.46 (1.02 to 2.10$)$ & 0.039 & 63 & $1.00(0.72$ to 1.39$)$ & 0.98 \\
\hline \multicolumn{8}{|c|}{ Grantham matrix score/sequence conservatione } \\
\hline Deleterious & 4 & 2 & $1.74(0.32$ to 9.64$)$ & 0.52 & 3 & $1.63(0.36$ to 7.33$)$ & 0.53 \\
\hline Intermediate (unclassified) & 46 & 22 & $1.75(1.02$ to 3.01$)$ & 0.043 & 25 & $1.15(0.69$ to 1.91$)$ & 0.60 \\
\hline Neutral & 99 & 40 & $1.44(0.95$ to 2.16$)$ & 0.083 & 44 & 0.95 (0.65 to 1.39$)$ & 0.78 \\
\hline \multicolumn{8}{|l|}{$B R C A 2^{\mathrm{g}}$} \\
\hline Normal/polymorphism (reference) & 279 & 87 & 1 & & 137 & 1 & \\
\hline Definitely disease-causing variant & 11 & 13 & $3.69(1.57$ to 8.68$)$ & 0.003 & 10 & $1.83(0.76$ to 4.42$)$ & 0.18 \\
\hline Unclassified variant & 462 & 162 & 1.07 (0.79 to 1.46$)$ & 0.66 & 213 & $0.93(0.72$ to 1.21$)$ & 0.59 \\
\hline \multicolumn{8}{|l|}{ Unclassified variant classification using } \\
\hline \multicolumn{8}{|l|}{ Allele frequency } \\
\hline High risk (LFUV) & 71 & 18 & $0.81(0.45$ to 1.45$)$ & 0.48 & 40 & 1.15 (0.74 to 1.78$)$ & 0.54 \\
\hline Low risk (HFUV) & 385 & 138 & 1.09 (0.79 to 1.50$)$ & 0.59 & 168 & $0.88(0.67$ to 1.16$)$ & 0.36 \\
\hline \multicolumn{8}{|l|}{ Polyphene } \\
\hline Probably damaging missense & 32 & 7 & $0.73(0.31$ to 1.74$)$ & 0.48 & 17 & 1.09 (0.58 to 2.03$)$ & 0.79 \\
\hline Possibly damaging missense & 108 & 28 & 0.79 (0.48 to 1.29$)$ & 0.34 & 42 & $0.78(0.52$ to 1.18$)$ & 0.24 \\
\hline Benign missense & 310 & 118 & 1.15 (0.83 to 1.60$)$ & 0.41 & 146 & $0.95(0.72$ to 1.26$)$ & 0.73 \\
\hline
\end{tabular}

Sequence conservatione 
Table 3 (Continued)

\begin{tabular}{|c|c|c|c|c|c|c|c|}
\hline High or moderate & 157 & 42 & 0.80 (0.52 to 1.23$)$ & 0.31 & 61 & 0.78 (0.55 to 1.12$)$ & 0.18 \\
\hline High & 96 & 30 & 0.92 (0.56 to 1.51$)$ & 0.75 & 41 & $0.86(0.57$ to 1.31$)$ & 0.48 \\
\hline Moderate & 61 & 12 & $0.61(0.31$ to 1.21$)$ & 0.15 & 20 & 0.66 (0.38 to 1.14$)$ & 0.13 \\
\hline Low & 292 & 113 & 1.18 (0.84 to 1.65$)$ & 0.33 & 142 & $0.98(0.74$ to 1.31$)$ & 0.91 \\
\hline \multicolumn{8}{|l|}{ Grantham matrix score ${ }^{\mathrm{e}}$} \\
\hline High $(>60)$ & 450 & 155 & 1.05 (0.77 to 1.43$)$ & 0.76 & 205 & 0.92 (0.71 to 1.20$)$ & 0.53 \\
\hline Low $(\leq 60)$ & 9 & 3 & $0.98(0.25$ to 3.85$)$ & 0.98 & 5 & 1.11 (0.37 to 3.39$)$ & 0.85 \\
\hline \multicolumn{8}{|c|}{ Grantham matrix score/sequence conservatione } \\
\hline Deleterious & 124 & 34 & $0.83(0.52$ to 1.31$)$ & 0.42 & 51 & $0.83(0.56$ to 1.22$)$ & 0.34 \\
\hline Intermediate (unclassified) & 66 & 14 & $0.67(0.35$ to 1.27$)$ & 0.22 & 28 & $0.87(0.53$ to 1.41$)$ & 0.56 \\
\hline Neutral & 259 & 107 & 1.24 (0.89 to 1.75$)$ & 0.21 & 124 & $0.97(0.72$ to 1.30$)$ & 0.82 \\
\hline
\end{tabular}

OR, odds ratio; $\mathrm{Cl}$, confidence interval; LFUV, low-frequency unclassified variants; HFUV, high-frequency unclassified variants. aTwenty-two and 55 women with unknown BRCA1 and BRCA2 status were included as a separate category for corresponding analyses. Numbers do not add up when further classifying unclassified variants (UVs) using the classification methods since the UV carriers who had missing values for higher-risk UV categories could not be categorized into high-risk or low-risk groups and thus were added to the unknown group. bFamily history of breast cancer or ovarian cancer is the outcome (no family history (reference), first degree, and second degree). Family history unknown cases were deleted from the analysis. All analyses were adjusted for age at diagnosis ( $<35$ years, 35 to $<40$ years, 40 to $<45$ years, $45+$ years). ${ }^{\circ}$ Based on the chi-square test. dWhen analyzing BRCA1, the model was further adjusted for the BRCA2 mutation status (definitely disease-causing variant, non-definitely disease-causing variant, unknown). eSplice/inframe deletion carriers of BRCA1 were excluded when analyzing BRCA1. Splice/ inframe deletion carriers of BRCA2 were excluded when analyzing BRCA2. fNumbers do not add up due to one additional subject classified after combining the high and moderate groups who was in the unknown BRCA1 status before combining. gWhen analyzing BRCA2, the model was further adjusted for BRCA1 mutation status (definitely disease-causing variant, non-definitely disease-causing variant, unknown).

for BRCA2 DDCV carriers is not as early as for BRCA1 DDCV carriers [29]. In our study, the median ages were 40 and 45 years for $B R C A 1$ and $B R C A 2$ DDCV carriers, respectively.

Homozygous deleterious mutations in BRCA1/BRCA2 are lethal [39-42]. In the present study, all of the low-risk UVs classified using the allele frequency method (except those that were common only in African-Americans) were observed as homozygous and therefore should be benign. Consistent with this, all our low-risk UVs (HFUVs) that have been classified by the BIC were assessed as clinically nonimportant. On the contrary, quite a few variants classified by the BIC as nonimportant are rare variants, and are therefore classified as high-risk UVs (LFUVs) in our study. If a variant has arisen very recently, its population frequency will be low even though the variant is not clinically important [43]. The allele-frequency method may therefore be better for the purpose of describing low-risk UVs than high-risk UVs.

The GMS is a pairwise comparison of the two substituted amino acids, and it has been argued that a multiple comparison - that is, a comparison of the substituted amino acids taking into account the natural variation of the substituted site across species - would provide better information $[44,45]$. One method of achieving such a multiple comparison is to use the integrated method of Abkevich and colleagues [26]. In our study, however, this method was not an improvement over the individual application of the two methods.
The Polyphen algorithm compares homologous sequences for conservation and examines the structural and physicochemical aspects of the substitution. We found that the high-risk UV carriers identified using Polyphen had the highest odds ratio of first-degree family history among those identified using all other methods. We also found that the number of clinically nonimportant variants that were classified as high risk or medium risk was smallest when using Polyphen. The Polyphen algorithm has been reported to have the smallest false-positive rate among the various online algorithms, including SIFT [35]. Polyphen has previously not been applied for BRCA1/BRCA2 whereas SIFT has been adopted for BRCA1 [24,25]. Our results suggest that Polyphen might be useful to identify highrisk UVs, especially when the UV has never been reported and/or clinical information is not available.

Efforts to classify UVs are accumulating: several groups have used simple combinations of sequence conservation and the severity of amino acid substitutions [24-26]. Whether the classification is clinically valid, however, has not been systematically examined [26]. Other studies have used extensive multifactorial models, most of them focusing on a few BRCA1 UVs. These models incorporate several approaches used in this study as well as clinical characteristics [46], co-occurrence with deleterious mutations $[19,46]$, and histopathological information [19]. While clinical and co-occurrence information has provided strong evidence to classify UVs $[37,46]$, however, such information is not always available, especially for UVs that have not been reported before. Further, 
Breast Cancer Research Vol 10 No 1 Lee et al.

it has been suggested that these "ideal" criteria cannot clas-

Table 4

Classification of BRCA1/BRCA2 variants (unclassified variants) that were considered clinically not important in the Breast Cancer Information Core database

\begin{tabular}{|c|c|c|}
\hline Unclassified variant classification method & Number of $B R C A 1$ variants & Number of $B R C A 2$ variants \\
\hline \multicolumn{3}{|l|}{ Allele frequency } \\
\hline High risk (LFUV) & $1(1200 \mathrm{H})$ & $\begin{array}{l}15 \text { (S326R, S384F, D596H, T598A, S976I, C1290Y, D1420Y, } \\
\text { G1529R, H2116R, T2515I, A2717S, V2728I, S2835P, E2856A, } \\
\text { T3013I) }\end{array}$ \\
\hline Low risk (HFUV) & $2($ (I379M, D693N) & $6(\mathrm{~N} 372 \mathrm{H}, \mathrm{N} 289 \mathrm{H}, \mathrm{L929S}, \mathrm{N987I}, \mathrm{N991D}, \mathrm{T} 1414 \mathrm{M})$ \\
\hline \multicolumn{3}{|l|}{ Polyphen } \\
\hline High risk (probable) & 0 & 3 (N987I, C1290Y, G1529R, H2116R) \\
\hline Medium risk (possible) & 1 (І379M) & $\begin{array}{l}8(\mathrm{~N} 289 \mathrm{H}, \mathrm{N} 372 \mathrm{H}, \mathrm{S} 384 \mathrm{~F}, \mathrm{D} 596 \mathrm{H}, \mathrm{S} 976 \mathrm{I}, \mathrm{D} 1420 \mathrm{Y}, \mathrm{T} 2515 \mathrm{I}, \\
\text { E2856A) }\end{array}$ \\
\hline Low risk (benign) & $2(\mathrm{D} 693 \mathrm{~N}, 1200 \mathrm{H})$ & $\begin{array}{l}9 \text { (S326R, T598A, L929S, N991D, T1414M, A2717S, V2728I, } \\
\text { S2835P, T3013I) }\end{array}$ \\
\hline \multicolumn{3}{|l|}{ Sequence conservation } \\
\hline High risk (high conservation) & 1 (I379M) & 4 (N289H, D596H, G1529R, E2856A) \\
\hline Medium risk (moderate conservation) & $1(\mathrm{Q} 1200 \mathrm{H})$ & $\begin{array}{l}10 \text { (S326R, T598A, V2728I, S384F, S976I, N987I, C1290Y, D1420Y, } \\
\text { H2116R, T2515I) }\end{array}$ \\
\hline Low risk (low conservation) & $1(\mathrm{D} 693 \mathrm{~N})$ & 7 (N372H, L929S, N991D, T1414M, A2717S, S2835P, T3013I) \\
\hline \multicolumn{3}{|l|}{ Grantham matrix score } \\
\hline High risk $(>60)$ & $1(\mathrm{Q} 1200 \mathrm{H})$ & $\begin{array}{l}17 \text { (N289H, S326R, N372H, S384F, D596H, L929S, S976l; C1290Y, } \\
\text { T1414M, D1420Y, G1529R, T2515l, A2717S, S2835P, N987I, } \\
\text { E2856A, T3013l) }\end{array}$ \\
\hline Low risk $(\leq 60)$ & $2($ (I379M, D693N) & 4 (T598A, N991D, H2116R, V2728I) \\
\hline \multicolumn{3}{|l|}{ Grantham matrix score/sequence conservation } \\
\hline High risk (deleterious) & 0 & 7 (N289H, D596H, L929S, N987I, 1420Y, G1529R, E2856A) \\
\hline Medium risk (unclassified) & $2($ (I379M, 1200H) & 7 (S326R, S384F, S976I, H2116R, T2515I, A2717S, T3013I) \\
\hline Low risk (neutral) & $1(\mathrm{D} 693 \mathrm{~N})$ & 7 (N372H, T598A, N991D, C1290Y, T1414M, V2728I, S2835P) \\
\hline
\end{tabular}

LFUV, low-frequency unclassified variants; HFUV, high-frequency unclassified variants.

sify the majority of the UVs [37]. The classification methods used in the present study may serve as "readily available" additional information to classify Uvs.

\section{Conclusion}

The present study suggests that the application of different methodologies such as allele frequency, Polyphen, the GMS, and sequence conservation may be useful for evaluating UVs, especially when little functional or clinical data are available. While we found high correlations between these classification methods, our study suggests that each method has different levels of false-positives and false-negatives. The Polyphen algorithm appeared more appropriate in identifying high-risk variants whereas the allele frequency may be useful in classifying high-frequency variants as nonimportant. Although our study does not directly address the question of whether each specific UV is associated with the risk of breast cancer, our results suggest that these methods could be helpful in under- standing the significance of a UV especially when other clinical or genetic information is not available. Further, the application of these methods may help to prioritize UVs for further functional or familial study.

\section{Competing interests}

The authors declare that they have no competing interests.

\section{Authors' contributions}

EL cleaned the data, classified BRCA1/BRCA2 unclassified variants, performed the data analysis and drafted the manuscript. RM-C participated in classification of the BRCA1/ $B R C A 2$ variants and revision of the manuscript. $\mathrm{HM}$ and $\mathrm{ZC}$ cleaned the data, and participated in classification of the $B R C A 1 / B R C A 2$ variants and revision of the manuscript. DVDB sequenced the BRCA1/BRCA2 genes, classified $B R C A 1 / B R C A 2$ variants, and participated in revision of the manuscript. LB participated in the design of the study and data 
collection, and revised the manuscript. BEH participated in the design and conception of the study, and supported the laboratory work. GU designed the study, supervised the data collection, participated in BRCA1/BRCA2 classification, supervised the data analysis, and revised the manuscript. All authors read and approved the final manuscript.

\section{Additional files}

The following Additional files are available online:

\section{Additional file 1}

Word file containing the detailed sequencing procedures and the classification approach integrating sequence conservation and the GMS.

See http://www.biomedcentral.com/content/

supplementary/bcr1865-S1.doc

\section{Additional file 2}

Word file containing a table listing the protein sequences used for cross-species comparison of BRCA1 and BRCA2.

See http://www.biomedcentral.com/content/

supplementary/bcr1865-S2.doc

\section{Additional file 3}

Word file containing a table listing all of the BRCA1/ BRCA2 variants identified in this study along with our classification of each variant in comparison with the classification according to the BIC database. See http://www.biomedcentral.com/content/ supplementary/bcr1865-S3.doc

\section{Acknowledgements}

The authors are deeply grateful to the interviewers of this study and to Ms Juliana Bamrick for managing the data collection. This study was supported by grants CA17054 and CA74847 from the National Cancer Institute, National Institutes of Health, by 4PB-0092 from the California Breast Cancer Research Program of the University of California, and in part through contract number N01-PC-35139. The collection of cancer incidence data used in this publication was supported by the California Department of Health Services as part of the statewide cancer reporting program mandated by California Health and Safety Code Section 103885. The ideas and opinions expressed herein are those of the authors, and no endorsement by the State of California, Department of Health Services is intended or should be inferred.

\section{References}

1. Miki $Y$, Swensen J, Shattuck-Eidens D, Futreal PA, Harshman K, Tavtigian S, Liu Q, Cochran C, Bennett LM, Ding W, Bell R, Rosenthal J, Hussey C, Tran T, McClure M, Frye C, Hattier T, Phelps R, Haugen-Strano A, Katcher H, Yakumo K, Gholami Z, Shaffer D, Stone S, Bayer S, Wray C, Bogden R, Dayananth P, Ward J, Tonin $\mathrm{P}$, et al.: A strong candidate for the breast and ovarian cancer susceptibility gene BRCA1. Science 1994, 266:66-71.
2. Wooster R, Neuhausen SL, Mangion J, Quirk Y, Ford D, Collins N, Nguyen K, Seal S, Tran T, Averill D, Fields P, Marshall G, Narod S, Lenoir GM, Lynch H, Feunteun J, Devilee P, Cornelisse CJ, Menko FH, Daly PA, Ormiston W, McManus R, Pye C, Lewis CM, CannonAlbright LA, Peto J, Ponder BAJ, Skolnick MH, Easton FN, Douglas $F$, et al:: Localization of a breast cancer susceptibility gene, BRCA2, to chromosome 13q12-13. Science 1994, 265:2088-2090.

3. Wooster R, Bignell G, Lancaster J, Swift S, Seal S, Mangion J, Collins N, Gregory S, Gumbs C, Micklem G: Identification of the breast cancer susceptibility gene BRCA2. Nature 1995, 378:789-792.

4. Hall JM, Lee MK, Newman B, Morrow JE, Anderson LA, Huey B King MC: Linkage of early-onset familial breast cancer to chromosome 17q21. Science 1990, 250:1684-1689.

5. Scully R, Chen J, Plug A, Xiao Y, Weaver D, Feunteun J, Ashley T, Livingston DM: Association of BRCA1 with Rad51 in mitotic and meiotic cells. Cell 1997, 88:265-275.

6. Moynahan ME, Chiu JW, Koller BH, Jasin M: Brca1 controls homology-directed DNA repair. Mol Cell 1999, 4:511-518.

7. Chapman MS, Verma IM: Transcriptional activation by BRCA1. Nature 1996, 382:678-679.

8. Anderson SF, Schlegel BP, Nakajima T, Wolpin ES, Parvin JD: BRCA1 protein is linked to the RNA polymerase II holoenzyme complex via RNA helicase A. Nat Genet 1998, 19:254-256.

9. Yarden Rl, Pardo-Reoyo S, Sgagias M, Cowan KH, Brody LC: BRCA1 regulates the G2/M checkpoint by activating Chk1 kinase upon DNA damage. Nat Genet 2002, 30:285-289.

10. Somasundaram $K$, Zhang $H$, Zeng $Y X$, Houvras $Y$, Peng $Y$, Zhang H, Wu GS, Licht JD, Weber BL, El-Deiry WS: Arrest of the cell cycle by the tumour-suppressor BRCA1 requires the CDKinhibitor p21WAF1/CiP1. Nature 1997, 389:187-190.

11. Williamson EA, Dadmanesh F, Koeffler HP: BRCA1 transactivates the cyclin-dependent kinase inhibitor p27(Kip1). Oncogene 2002, 21:3199-3206.

12. Chen J, Silver DP, Walpita D, Cantor SB, Gazdar AF, Tomlinson G, Couch FJ, Weber BL, Ashley T, Livingston DM, Scully R: Stable interaction between the products of the BRCA1 and BRCA2 tumor suppressor genes in mitotic and meiotic cells. Mol Cell 1998, 2:317-328.

13. Yu VP, Koehler M, Steinlein C, Schmid M, Hanakahi LA, van Gool AJ, West SC, Venkitaraman AR: Gross chromosomal rearrangements and genetic exchange between nonhomologous chromosomes following BRCA2 inactivation. Genes Dev 2000, 14:1400-1406.

14. Moynahan ME, Pierce AJ, Jasin M: BRCA2 is required for homology-directed repair of chromosomal breaks. Mol Cell 2001, 7:263-272.

15. Davies AA, Masson JY, Mcllwraith MJ, Stasiak AZ, Stasiak A, Venk itaraman AR, West SC: Role of BRCA2 in control of the RAD51 recombination and DNA repair protein. Mol Cell 2001, 7:273-282.

16. Yoshida K, Miki Y: Role of BRCA1 and BRCA2 as regulators of DNA repair, transcription, and cell cycle in response to DNA damage. Cancer Sci 2004, 95:866-871.

17. Breast Cancer Information Core Database [http:// research.nhgri.nih.gov/projects/bic]

18. Shattuck-Eidens D, Oliphant A, McClure M, McBride C, Gupte J, Rubano T, Pruss D, Tavtigian SV, Teng DH, Adey N, Staebell M, Gumpper K, Lundstrom R, Hulick M, Kelly M, Holmen J, Lingenfelter B, Manley S, Fujimura F, Luce M, Ward B, Cannon-Albright L, Steele L, Offit K, Gilewski T, Norton L, Brown K, Schulz C, Hampel $\mathrm{H}$, Schluger A, et al:: BRCA1 sequence analysis in women at high risk for susceptibility mutations. Risk factor analysis and implications for genetic testing. JAMA 1997, 278:1242-1250.

19. Chenevix-Trench $G$, Healey $S$, Lakhani $S$, Waring $P$, Cummings $M$, Brinkworth R, Deffenbaugh AM, Burbidge LA, Pruss D, Judkins T, Scholl T, Bekessy A, Marsh A, Lovelock P, Wong M, Tesoriero A, Renard H, Southey M, Hopper JL, Yannoukakos K, Brown M, Easton D, Tavtigian SV, Goldgar D, Spurdle AB: Genetic and histopathologic evaluation of BRCA1 and BRCA2 DNA sequence variants of unknown clinical significance. Cancer Res 2006, 66:2019-2027.

20. Wooster R, Weber BL: Breast and ovarian cancer. N Engl J Med 2003, 348:2339-2347.

21. Vallon-Christersson J, Cayanan C, Haraldsson K, Loman N, Bergthorsson JT, Brondum-Nielsen K, Gerdes AM, Moller P, Krist- 
offersson $\mathrm{U}$, Olsson $\mathrm{H}$, Borg A, Monteiro AN: Functional analysis of BRCA1 C-terminal missense mutations identified in breast and ovarian cancer families. Hum Mol Genet 2001, 10:353-360.

22. Phelan CM, Dapic V, Tice B, Favis R, Kwan E, Barany F, Manoukian $S$, Radice $P$, van der Luijt RB, van Nesselrooij BP, Chenevix-Trench G, kConFab , Caldes T, de la Hoya M, Lindquist S, Tavtigian SV, Goldgar D, Borg A, Narod SA, Monteiro AN: Classification of BRCA1 missense variants of unknown clinical significance. J Med Genet 2005, 42:138-146.

23. Ramensky V, Bork $P$, Sunyaev $S$ : Human non-synonymous SNPs: server and survey. Nucleic Acids Res 2002, 30:3894-3900

24. Fleming MA, Potter JD, Ramirez CJ, Ostrander GK, Ostrander EA: Understanding missense mutations in the BRCA1 gene: An evolutionary approach. Proc Natl Acad Sci USA 2003, 100:1151-1156.

25. Burk-Herrick A, Scally M, Amrine-Madsen H, Stanhope MJ, Springer MS: Natural selection and mammalian BRCA1 sequences: elucidating functionally important sites relevant to breast cancer susceptibility in humans. Mamm Genome 2006, $17: 257-270$.

26. Abkevich V, Zharkikh A, Deffenbaugh AM, Frank D, Chen $Y$, Shattuck D, Skolnick MH, Gutin A, Tavtigian SV: Analysis of missense variation in human BRCA1 in the context of interspecific sequence variation. J Med Genet 2004, 41:492-507.

27. Grantham R: Amino acid difference formula to help explain protein evolution. Science 1974, 185:862-864.

28. Berry DA, Iversen ES Jr, Gudbjartsson DF, Hiller EH, Garber JE, Peshkin BN, Lerman C, Watson P, Lynch HT, Hilsenbeck SG, Rubinstein WS, Hughes KS, Parmigiani G: BRCAPRO validation, sensitivity of genetic testing of BRCA1/BRCA2, and prevalence of other breast cancer susceptibility genes. $J$ Clin Oncol 2002, 20:2701-2712.

29. Eerola $\mathrm{H}$, Heikkila $\mathrm{P}$, Tamminen A, Aittomaki $\mathrm{K}$, Blomqvist $\mathrm{C}$, Nevanlinna $\mathrm{H}$ : Histopathological features of breast tumours in BRCA1, BRCA2 and mutation-negative breast cancer families. Breast Cancer Res 2005, 7:R93-R100.

30. Ma H, Bernstein L, Ross RK, Ursin G: Hormone-related risk factors for breast cancer in women under age $\mathbf{5 0}$ years by estrogen and progesterone receptor status: results from a casecontrol and a case-case comparison. Breast Cancer Res 2006, 8:R39.

31. Marchbanks PA, McDonald JA, Wilson HG, Folger SG, Mandel MG, Daling JR, Bernstein L, Malone KE, Ursin G, Strom BL, Norman SA, Wingo PA, Burkman RT, Berlin JA, Simon MS, Spirtas R, Weiss LK: Oral contraceptives and the risk of breast cancer. $N$ Engl J Med 2002, 346:2025-2032.

32. McKean-Cowdin R, Spencer Feigelson H, Xia LY, Pearce CL, Thomas DC, Stram DO, Henderson BE: BRCA1 variants in a family study of African-American and Latina women. Hum Genet 2005, 116:497-506

33. Freedman ML, Penney KL, Stram DO, Le Marchand L, Hirschhorn JN, Kolonel LN, Altshuler D, Henderson BE, Haiman CA: Common variation in BRCA2 and breast cancer risk: a haplotype-based analysis in the Multiethnic Cohort. Hum Mol Genet 2004, 13:2431-2441.

34. PolyPhen [http://coot.embl.de/PolyPhen]

35. Ng PC, Henikoff S: Predicting the effects of amino acid substitutions on protein function. Annu Rev Genomics Hum Genet 2006, 7:61-80

36. Thompson JD, Higgins DG, Gibson TJ: CLUSTAL W: improving the sensitivity of progressive multiple sequence alignment through sequence weighting, position-specific gap penalties and weight matrix choice. Nucleic Acids Res 1994, 22:4673-4680.

37. Easton DF, Deffenbaugh AM, Pruss D, Frye C, Wenstrup RJ, AllenBrady K, Tavtigian SV, Monteiro AN, Iversen ES, Couch FJ, Goldgar DE: A systematic genetic assessment of 1,433 sequence variants of unknown clinical significance in the BRCA1 and BRCA2 breast cancer-predisposition genes. $A m \mathrm{~J}$ Hum Genet 2007, 81:873-883.

38. Collaborative Group on Hormonal Factors in Breast Cancer: Familial breast cancer: collaborative reanalysis of individual data from 52 epidemiological studies including 58,209 women with breast cancer and 101,986 women without the disease. Lancet 2001, 358:1389-1399.
39. Ludwig T, Chapman DL, Papaioannou VE, Efstratiadis A: Targeted mutations of breast cancer susceptibility gene homologs in mice: lethal phenotypes of Brca1, Brca2, Brca1/Brca2, Brca1/ p53, and Brca2/p53 nullizygous embryos. Genes Dev 1997, 11:1226-1241.

40. Kuschel B, Gayther SA, Easton DF, Ponder BA, Pharoah PD: Apparent human BRCA1 knockout caused by mispriming during polymerase chain reaction: implications for genetic testing. Genes Chromosomes Cancer 2001, 31:96-98.

41. Gowen LC, Johnson BL, Latour AM, Sulik KK, Koller BH: Brca1 deficiency results in early embryonic lethality characterized by neuroepithelial abnormalities. Nat Genet 1996, 12:191-194.

42. Sharan SK, Morimatsu M, Albrecht U, Lim DS, Regel E, Dinh C, Sands A, Eichele G, Hasty P, Bradley A: Embryonic lethality and radiation hypersensitivity mediated by Rad51 in mice lacking Brca2. Nature 1997, 386:804-810.

43. Thompson EA, Neel JV: Allelic disequilibrium and allele frequency distribution as a function of social and demographic history. Am J Hum Genet 1997, 60:197-204.

44. Tavtigian SV, Deffenbaugh AM, Yin L, Judkins T, Scholl T, Samollow PB, de Silva D, Zharkikh A, Thomas A: Comprehensive statistical study of 452 BRCA1 missense substitutions with classification of eight recurrent substitutions as neutral. J Med Genet 2006, 43:295-305.

45. Tavtigian SV, Samollow PB, de Silva D, Thomas A: An analysis of unclassified missense substitutions in human BRCA1. Fam Cancer 2006, 5:77-88.

46. Goldgar DE, Easton DF, Deffenbaugh AM, Monteiro AN, Tavtigian SV, Couch FJ, Breast Cancer Information Core Steering Committee: Integrated evaluation of DNA sequence variants of unknown clinical significance: application to BRCA1 and BRCA2. Am J Hum Genet 2004, 75:535-544. 